\title{
Suspended Sediment Modeling with Continuously Lagging Input Variables Using Artificial Intelligence and Physics based Models
}

\author{
Daniel Prakash Kushwaha* and Devendra Kumar
}

Department of Soil and Water Conservation Engineering, College of Technology, G. B. Pant University of Agriculture and Technology, Pantnagar-263145, Uttarakhand, India

*Corresponding author

\section{A B S T R A C T}

\begin{tabular}{|l|}
\hline K e y w o r d s \\
Sediment rating curve, \\
Multiple linear \\
regression, Artificial \\
neural network, \\
Minimum description \\
length, Akaike's \\
information criterion.
\end{tabular}

Artificial neural network (ANN) models were developed to predict daily suspended sediment concentration (SSC) for the Baitarani River at Champua station using daily SSC and daily discharge. ANN models were calibrated by using multilayer feed forward back propagation neural networks with sigmoid activation function and Levenberg-Marquardt (L-M) learning algorithm. The performance of the developed models was evaluated qualitatively and quantitatively. In qualitative evaluation of models, observed suspended sediment concentration (OSCC) and computed suspended sediment concentration (CSSC) were compared using sediment hydrographs and scatter plots during testing period. Akaike's information criterion (AIC), correlation coefficient ( $r$ ), mean square error (MSE), root mean square error (RMSE), minimum description length (MDL), coefficient of efficiency (CE) and normalized mean square error (NMSE) indices were used for quantitative performance evaluation of the models. Results indicate that M-6 model with (7-5-5-1) network architecture is better than all models and it was also found that ANN based model is better than physics based models such as sediment rating curve and multiple linear regression for the prediction of SSC.

\section{Introduction}

Modelling of soil erosion rate has become very essential in large catchments in which all the watersheds must be treated and developed on priority basis to minimize the soil loss and increase their productivity. On the basis of such governing processes, following models were categorised into two components i.e. physics based models and other one is system based theoretic models. In Physics based models hydrologic behaviour of watershed is modelled through different physical process. Number of physics based models or phenomenon were proposed by researchers to evaluate and predict sediment yielded.
Until now, many physics based models have been employed for prediction of sediment yield, using empirical formulae, sediment rating curve (SRC), various statistical techniques, multiple linear regression (MLR), USLE, MUSLE, RUSLE etc. Researchers found it difficult to arrive at a fixed conclusion as mechanism of sediment load transportation and non-linear behaviour of selected hydrologic parameters of that study was difficult to model. In system theoretic models, input data was transferred to the output zone with the help of transfer functions as they do not include any physical 
characteristics of input parameters. A great revolution has been observed in prediction and resolving hydrologic problems by various researchers when ANN was used as tool with any system theoretic model. Main character of black box was to simulate complex natural process and therefore it was considered of much significance when it was employed to solve different types of water resource problems.

ANN, one of the most popular soft computing techniques, is example of system theoretic models. Details of watershed characteristics are not required in the application of ANN and results obtained are closer to the observed ones because ANN uses linear and non-linear concepts both in the development of model and can be performed with memory less or dynamic system. ANN has two main merits, first one is to perform action without any dependence on past events and second one is to overcome any inconsistency in data taken as input or if there is less availability of data.

In recent years, ANN based system theoretic models have been employed in solving hydrological and meteorological problems such as rainfall runoff modelling, runoff sediment modelling (Singh et al., 2013; Rai and Mathur, 2008; Kisi et al., 2012; Gharde et al., 2015; Jain, 2001; Kermani et al., 2016; Kumar et al., 2016; Olyaie et al., 2015; Ghorbani et al., 2013; Eisazadeh et al., 2013; Shabani et al., 2012; Kumar et al., 2011; Kisi, 2010), river flow estimation (Nayak et al., 2004), evapotranspiration process (Kuo et al., 2011; Khoob, 2008), optimization of water supply system etc.

The main purpose of the present study is development, validation and performance evaluation of ANN models to estimate concentration of suspended sediment on daily basis with continuously lagging input variables at Champua station located at the top of the Baitarani river basin falling in the state of Odisha, India and comparison of best selected ANN model with the physics based models such as SRC and MLR.

\section{Materials and Methods}

\section{Study area and data collection}

The Baitarani River (Fig. 1) originates from the Guptaganga hills ranges near Mankarancho village and flows eastward and joins the Bay of Bengal. The total area of Baitarani river basin is $10982 \mathrm{sq}$. $\mathrm{km}$ The Baitarani river basin is located between $85^{\circ} 10^{\prime}$ to $87^{0} 03^{\prime}$ east longitudes and between $20^{0} 35^{\prime}$ to $22^{0} 15^{\prime}$ north latitudes. Most of the rainfall in the watershed is received from the South-West monsoons from June to September (about 80\%) and average rainfall is $1187 \mathrm{~mm}$. Monsoon season data of SSC and discharge from Champua gauging station of Central Water Commission (CWC) from 1977 to 2006 have been used in this study and data variation at each day has been shown in Figures 2 and 3.

\section{MLR}

In MLR equation, relationship between dependent variable and several independent variables is established by fitting them in a linear equation. Regression analysis is commonly used to describe quantitative relationships between a dependent variable and one or more independent variables (Shirsath and Singh, 2010). MLR are used to model linear variables based on a least squares technique. However, MLR present some shortcomings and drawbacks in predicting nonlinear situations, given their nature of capturing strictly linear relations.

$Y=b_{0}+b_{1} X_{1}+b_{2} X_{2}+$

$\cdots+b_{n} X_{n}$ 
Where, $Y$ is the dependent variable, $b_{0}, b_{1}, b_{2}$, $b_{n}$ are the regression coefficients for the linear equation and $X_{1}, X_{2}, \ldots \ldots X_{n}$ are the independent variables.

\section{SRC}

The SRC, generally follow the following form of relationship given below;

$S_{t}=a\left(Q_{t}\right)^{b} \ldots(2)$

Where, $a$ and $b$ are the coefficients, $S_{t}$ is present day SSC and $Q_{t}$ is present day discharge. The values of $a$ and $b$ for a particular stream are determined from data via a linear regression between $\log S_{t}$ and $\log Q_{t}$.

A major limitation of this approach is that it is not able to consider the hysteresis effect. In this study, the values of $a$ and $b$ are computed by using the least squares method (Jain, 2008; Rajaee et al., 2009).

\section{ANN}

The original concept of an ANN or neural network was developed by Warren McCulloch and Walter Pitts (1943). ANN is built from number of different nonlinear processing elements which are known as "neurons" each of which receives connections from other neurons or and itself according to the training algorithm.

The signals which are flowing on the connections are scaled by adjustable parameters known as weights (Principe et al., 2000). ANN works are inspired by structure of human brain and it is well suited for complicated task in hydrologic system too such as evaporation modelling, rainfall modelling, river flow modelling, suspended sediment yield modelling etc. ANN has been proven to provide better solution for estimation of SSC.

\section{Structural description of ANN}

ANN structures, broadly classified as recurrent (involving feedback) or non-recurrent (without feedback), have number of processing elements (also called neurons, neuroses) and connections. The basic structure of ANN consists of an input layer, hidden layer/layers and an output layer. In multilayer feed forward ANN, one or more hidden layers are present and this neural network architecture has been used in this study.

The function of the ANN is to map a set of inputs to a set of outputs. A perceptron is shown in Figure 4. Let $x_{i}(i=1,2, \ldots, \mathrm{m})$ are inputs and $w_{i}(i=1,2, \ldots, \mathrm{m})$ are respective weights. The net input to the node is given as;

$$
\text { net }=\sum_{i=1}^{n} x_{i} w_{i} \ldots \text { (3) }
$$

The net input then goes through activation function $f$ and then the output $y$ of the node is computed as;

$y=f($ net $)$

The error calculated at the output layer is sent back to the hidden layers and then passed on to the input layer, so that updates for the connection weights are determined the sum square error $E$, is given by;

$E=\frac{1}{2} \sum_{k=1}^{n o}\left(y_{k}-t_{k}\right)^{2} \ldots(5)$

Where, $t_{k}$ is the observed output or output desired at the $k^{\text {th }}$ neuron and $y_{k}$ is the calculated output at the same neuron. Weights are updated and changed from their old values to minimize the error. The learning process starts with a random set of weights. Weights are updated through error back-propagation during the training process in each iteration, 
in order to reach better efficient weights and speed of learning process it arise many types of back propagation.

\section{Learning algorithms}

The learning algorithm is used to calculate the adjusted weights and biases of the network so that the error between computed and observed output is minimized. In more recent studies the Levenberg-Marquardt (L-M) algorithm is being used due to its higher efficiency and high convergence Speed. L-M algorithm was shown to be more efficient than the CG and GDX algorithms (Solaimani, 2009). Many other researchers have also proved the efficiency of L-M algorithm. A specialized back propagation optimization technique known as L-M Back propagation developed by Hagan and Menhaj (1994) was used in the study to train the MLP-ANN.

\section{Model Development}

\section{Identification of input and output variables}

The current day suspended sediment is not only dependent on the stream flow of the current time but also on the runoff and suspended sediment of the previous periods (Cobaner et al., 2009).

Several combinations of the discharge and SSC were tried to construct the proper input structure.

\section{Development of ANN models}

After the identification of input and output variables, various ANN models were developed for the station under consideration (Table 1).

Where, $S_{t}$ is d present day SSC, $S_{(t-1)}$ is the one day lag SSC, $S_{(t-2)}$ is the two days lag SSC, $S_{(t-3)}$ is the three days lag SSC and $S_{(t-4)}$ is the four days lag SSC, $Q_{t}$ is the present day discharge, $Q_{(t-1)}$ is the one day lag discharge, $Q_{(t-2)}$ is the two days lag discharge, $Q_{(t-3)}$ is the three days lag discharge and $Q_{(t-4)}$ is the four days lag discharge.

\section{Training and testing of MLP-ANN models}

Data accounting from year 1977 to 1996 was used for model calibration and data accounting from year 1997 and 2006 was used for model validation. In this study, the training of ANN models were done by using single and double hidden layers neural networks, processing elements from 1 to 10 in both the hidden layers simultaneously, sigmoid activation function, Levenberg Marquardt learning rule, maximum 1000 number of epochs and 0.001 training threshold assigned as per ANN model.

\section{Performance evaluation of models}

In this study, sediment hydrographs and scatter plots are used for qualitative performance evaluation of models and the different performance evaluating indices were used for quantitative performance evaluation of models and discussed below;

\section{Normalized Mean Square Error (NMSE)}

$$
\text { NMSE }=\frac{P N M S E}{\sum_{j=0}^{P} \frac{N \sum_{i=0}^{N} S_{c i j}^{2}-\left(\sum_{i=0}^{N} S_{c i j}\right)^{2}}{N}}
$$

\section{Root mean square error (RMSE)}

$$
R M S E=\sqrt{\frac{\sum_{i=i}^{N}\left(S_{c i}-S_{o i}\right)^{2}}{N}} \ldots(7)
$$

\section{Correlation coefficient (r)}

$$
r=\frac{\sum_{i=1}^{N}\left(S_{o i}-S_{o m}\right) \sum_{i=1}^{N}\left(S_{c i}-S_{c m}\right)}{\sqrt{\sum_{i=1}^{N}\left(S_{o i}-S_{o m}\right)^{2}} \sqrt{\sum_{i=1}^{N}\left(S_{c i}-S_{c m}\right)^{2}} \ldots}
$$




\section{Minimum Description Length (MDL)}

Rissanen's minimum description length (MDL), similar to the AIC, combines the error of model with the number of degree of freedom to find out the level generalization. The goal here is to minimize this term.

$M D L(k)=N \ln (M S E)+0.5 k \ln (N) . .(9)$

Where, $k$ is the number of network weights, $N$ is the total number of observations in the training/testing data set.

\section{Akaike's Information Criterion (AIC)}

Akaike's information criterion (AIC) measures the trade off between training performance of the model and network size. The goal in this case is to minimize this term so that a network with the best generalization is produced.

\section{$\operatorname{AIC}(k)=2 k+N \ln (M S E) \ldots(10)$}

Where, $k$ is the number of network weights, $N$ is the total number of observations in the training or testing data set and MSE is the mean square error.

\section{Coefficient of efficiency (CE)}

$C E=1-\frac{\sum_{i=1}^{N}\left(S_{o i}-S_{c i}\right)^{2}}{\sum_{i=1}^{N}\left(S_{o i}-S_{o m}\right)^{2}} \ldots$

Where, $S_{c i}$ and $S_{o i}$ are the computed and measured SSC for $i^{\text {th }}$ exemplar, $S_{o m}$ and $S_{c m}$ are the mean of CSSC and OSSC values., $N$ is the total number of observations in the training or testing data set, $k$ is the number of network weights, $P$ is the number of output processing elements, $S_{c i j}$ is the computed output for $i^{\text {th }}$ observations and at $j^{\text {th }}$ processing element.

\section{Results and Discussion}

Qualitative performance evaluation of daily SSC models

In this study, various ANN architectures were applied using trial and error procedure and network architecture which were found to be best during testing and training using qualitative evaluation (Table 2).

The OSSC and CSSC for ANN based models were compared graphically using sediment hydrographs and scatter plots during testing period because during training period the model performance can be improved by over fitting the data and that cannot be consider under selection of best models but model performance during testing period is independent of this.

\section{Performance evaluation based on sediment hydrographs}

Sediment hydrographs for qualitative evaluation are plotted between OSSC and CSSC values on ordinate and their corresponding occurrence time on abscissa (Fig. 5) It was observed from sediment hydrographs that out of eight models, M-3, M-6 and M-7 very closely predict the peaks accurately and rest of the models i.e. M-1, M2, M-4, M-5 and M-8 over predict the peaks.

\section{Performance evaluation based on scatter plots}

Scatter plots are plotted between CSSC values on ordinate and their corresponding OSSC values on the abscissa (Fig. 6). The observations of scatter diagrams on the basis of best fit line and 1:1 line indicate that the SSC are over predicted for smaller values of SSC and under predicted for larger values of SSC using M-1, M-3, M-4, M-5, M-6 and M7 models and over predicting for M-2 and M- 
8 models. It was also observed for M-3 model that most of the SSC values are under predicted and very few SSC values are over predicted.

M-6 and M-7 models nicely demonstrate that most of the data points are quite near to line of best fit in comparison to other models. But model M-7 shows some deviations from best fit line in comparison to M-6 model due to which performance of the M-7 model was found better than other models but inferior than M-6. Therefore, M-6 model was found to be better than other models for daily SSC prediction. The values of coefficient of determination $\left(\mathrm{R}^{2}\right)$ for models $\mathrm{M}-1, \mathrm{M}-2, \mathrm{M}-$ 3, M-4, M-5, M-6, M-7 and M-8 are 0.588, $0.656,0.727,0.692,0.6830 .903,0.876$ and 0.579 , respectively.

Quantitative performance evaluation of daily SSC models

Quantitative evaluation is considered to be effective in performance evaluation of the models and free from personal bias which occurs in qualitative evaluation. The values of indices for testing period for all the models are given in Table 3. The models having minimum values of RMSE, NMSE, MDL and AIC and higher values of $\mathrm{CE}$ and $r$ were considered as best models. Based on the selected criteria, five ANN based models i.e. M-3, M-4, M-5, M-6 and M-7 were found to be performing better than out of the eight models.

Based on comparison among five selected models i.e. M-3, M-4, M-5, M-6 and M-7, M6 model had the minimum values of NMSE (0.1013), AIC (-11075.64), MDL (-10949.82) and RMSE $(0.0100 \mathrm{~g} / \mathrm{l})$ and maximum values of CE (0.9937) and $r$ (0.951). The order of the models performance from best to worst was found to be M-6 > M-7 > M-3 > M-4 > M-5. Therefore, performance of the M-6 model was found to be best in daily SSC.
On the basis of comparison between qualitative and quantitative evaluation for best model, it was found that M-6 model in which present day SSC depends on the present day discharge, one-two and three lag days discharge, one-two and three lag days SSC with (7-5-5-1) network architecture i.e. 7 input variables, five-five neurons in first and second hidden layer and single output processing element is best out of eight models.

\section{Qualitative comparison of best ANN-MLP model with physics based models}

The OSSC and CSSC for ANN based models were compared graphically with the results of MLR analysis and SRC methods using sediment hydrographs and scatter plots during testing period.

\section{Comparison based on sediment hydrographs}

M-6 model of ANN-MLP nicely demonstrates that most of the data points are quite near the line of best fit in comparison to other methods. In Figure 7, it was observed from sediment hydrograph of ANN-MLP, that it is very closely predicting the peaks accurately out of three sediment hydrographs and for MLR analysis and SRC, these are over predicting the peaks. It was also observed that sediment hydrograph of MLR analysis is giving better result than SRC.

\section{Comparison based on scatter plots}

In Figure 8, the observations of scatter diagrams on the basis of best fit line and 1:1 line (dotted line) indicate that the SSC are over predicted for smaller values of SSC and under predicted for larger values of SSC for all the methods applied above for M-6 model.

It was also observed that most of the SSC values are under predicted and very few SSC 
values are over predicted for ANN-MLP. Therefore, ANN-MLP was found to be better than other methods for daily SSC prediction. The values of $R^{2}$ for ANN-MLP, MLR and SDR are $0.903,0.438$ and 0.313 , respectively, for M-6 model.

\section{Quantitative comparison of best ANN-} MLP model with physics based models

The values of indices for testing period for all the methods are given in Table 4. The methods having minimum values of RMSE, higher values of CE and $r$ were considered as best methods. Based on the above criteria, ANN-MLP was found to be performing better than MLR and SDR.

Based on comparison among ANN-MLP, MLR and SDR for M-6 model, ANN-MLP based M-6 model has the minimum value of RMSE $(0.0100 \mathrm{~g} / \mathrm{l})$ and maximum values of CE (0.9937) and $r(0.951)$. The order of the methods from best to worst was found to be ANN-MLP > MLR > SDR. On the basis of comparison between qualitative and quantitative evaluation for best method, it was found that ANN-MLP method is best out of MLR and SDR. Finally, ANN-MLP based M6 model was found better than all the models and methods in this study for the prediction of daily SSC.

ANN based models were developed to predict daily SSC for the Baitarani River at Champua station using daily discharge and daily SSC. The 30 years data (June 1977 to September 2006) used in this study was divided into two sets viz. a training set (1977-1996) for model calibration and a testing set (1997-2006) for validation of models. Eight models were developed by using various combinations of discharge and SSC and the performance of the developed models was evaluated qualitatively by visual observations and quantitatively. Furthermore, a comparison was made between ANN-MLP, MLR and SDR methods for best selected model. There are following conclusions are drawn from the results of the study;

Table.1 List of various ANN models

\begin{tabular}{cl}
\hline Model & Output-input variables \\
\hline M-1 & $S_{t}=f\left(S_{(t-1)}, Q_{t}\right)$ \\
M-2 & $S_{t}=f\left(S_{(t-1)}, Q_{t}, Q_{(t-1)}\right)$ \\
M-3 & $S_{t}=f\left(S_{(t-1)}, S_{(t-2)}, Q_{t}, Q_{(t-1)}\right)$ \\
M-4 & $S_{t}=f\left(S_{(t-1)}, S_{(t-2),}, Q_{t}, Q_{(t-1)}, Q_{(t-2)}\right)$ \\
M-5 & $S_{t}=f\left(S_{(t-1)}, S_{(t-2),}, S_{(t-3),}, Q_{t}, Q_{(t-1)}, Q_{(t-2)}\right)$ \\
M-6 & $S_{t}=f\left(S_{(t-1)}, S_{(t-2)}, S_{(t-3)}, Q_{t}, Q_{(t-1)}, Q_{(t-2)}, Q_{(t-3)}\right)$ \\
M-7 & $S_{t}=f\left(S_{(t-1)}, S_{(t-2),}, S_{(t-3)}, S_{(t-4)}, Q_{t}, Q_{(t-1)}, Q_{(t-2)}, Q_{(t-3)}\right)$ \\
M-8 & $S_{t}=f\left(S_{(t-1)}, S_{(t-2)}, S_{(t-3)}, S_{(t-4)}, Q_{t}, Q_{(t-1)}, Q_{(t-2)}, Q_{(t-3)}, Q_{(t-4)}\right)$ \\
\hline
\end{tabular}

Table.2 Best selected network architecture for ANN models

\begin{tabular}{ccc}
\hline Model & Network architecture & No. of epochs ran \\
\hline M-1 & $2-10-10-1$ & 296 \\
M-2 & $3-10-10-1$ & 245 \\
M-3 & $4-10-10-1$ & 127 \\
M-4 & $5-6-6-1$ & 196 \\
M-5 & $6-10-10-1$ & 51 \\
M-6 & $7-5-5-1$ & 84 \\
M-7 & $8-6-6-1$ & 74 \\
M-8 & $9-10-10-1$ & 71 \\
\hline
\end{tabular}


Table.3 Performance evaluation indices of ANN-MLP models for testing period

\begin{tabular}{cccccccc}
\hline \multirow{2}{*}{ Model } & Network & \multicolumn{5}{c}{ Testing } \\
\cline { 3 - 7 } & architecture & RMSE $(\mathrm{g} / \mathrm{l})$ & $\mathrm{r}$ & $\mathrm{CE}$ & NMSE & AIC & MDL \\
\hline M-1 & $2-10-10-1$ & 0.0251 & 0.780 & 0.9604 & 0.6424 & -8661.94 & -8411.86 \\
M-2 & $3-10-10-1$ & 0.0224 & 0.831 & 0.9685 & 0.5066 & -8931.54 & -8665.93 \\
M-3 & $\mathbf{4 - 1 0 - 1 0 - 1}$ & $\mathbf{0 . 0 1 7 6}$ & $\mathbf{0 . 8 5 3}$ & $\mathbf{0 . 9 8 0 5}$ & $\mathbf{0 . 3 1 7 3}$ & $\mathbf{- 9 4 8 2 . 5 6}$ & $\mathbf{- 9 2 0 1 . 4 1}$ \\
M-4 & $\mathbf{5 - 6 - 6 - 1}$ & $\mathbf{0 . 0 1 9 2}$ & $\mathbf{0 . 8 5 2}$ & $\mathbf{0 . 9 7 6 7}$ & $\mathbf{0 . 3 7 8 0}$ & $\mathbf{- 9 4 4 8 . 7 8}$ & $\mathbf{- 9 3 0 7 . 4 3}$ \\
M-5 & $\mathbf{6 - 1 0 - 1 0 - 1}$ & $\mathbf{0 . 0 2 0 5}$ & $\mathbf{0 . 8 5 0}$ & $\mathbf{0 . 9 7 3 6}$ & $\mathbf{0 . 4 2 6 2}$ & $\mathbf{- 9 0 8 2 . 5 6}$ & $\mathbf{- 8 7 7 0 . 3 5}$ \\
M-6 & $\mathbf{7 - 5 - 5 - 1}$ & $\mathbf{0 . 0 1 0 0}$ & $\mathbf{0 . 9 5 1}$ & $\mathbf{0 . 9 9 3 7}$ & $\mathbf{0 . 1 0 1 3}$ & $\mathbf{- 1 1 0 7 5 . 6 4}$ & $\mathbf{- 1 0 9 4 9 . 8 2}$ \\
M-7 & $\mathbf{8 - 6 - 6 - 1}$ & $\mathbf{0 . 0 1 1 4}$ & $\mathbf{0 . 9 3 7}$ & $\mathbf{0 . 9 9 1 8}$ & $\mathbf{0 . 1 2 8 7}$ & $\mathbf{- 1 0 7 2 6 . 9 2}$ & $\mathbf{- 1 0 5 5 7 . 6 1}$ \\
M-8 & $9-10-10-1$ & 0.0283 & 0.775 & 0.9497 & 0.8057 & -8245.65 & -7886.83 \\
\hline
\end{tabular}

Table.4 Quantitative comparison of ANN-MLP based M-6 model during testing period

\begin{tabular}{cccc}
\hline Method & RMSE $(\mathrm{g} / \mathrm{l})$ & $\mathrm{r}$ & $\mathrm{CE}$ \\
\hline ANN-MLP & $\mathbf{0 . 0 1 0 0}$ & $\mathbf{0 . 9 5 1}$ & $\mathbf{0 . 9 9 3 7}$ \\
MLR & 0.1037 & 0.662 & 0.3233 \\
SRC & 0.1532 & 0.559 & -0.4772 \\
\hline
\end{tabular}

Fig.1 Location map of Baitarani river basin

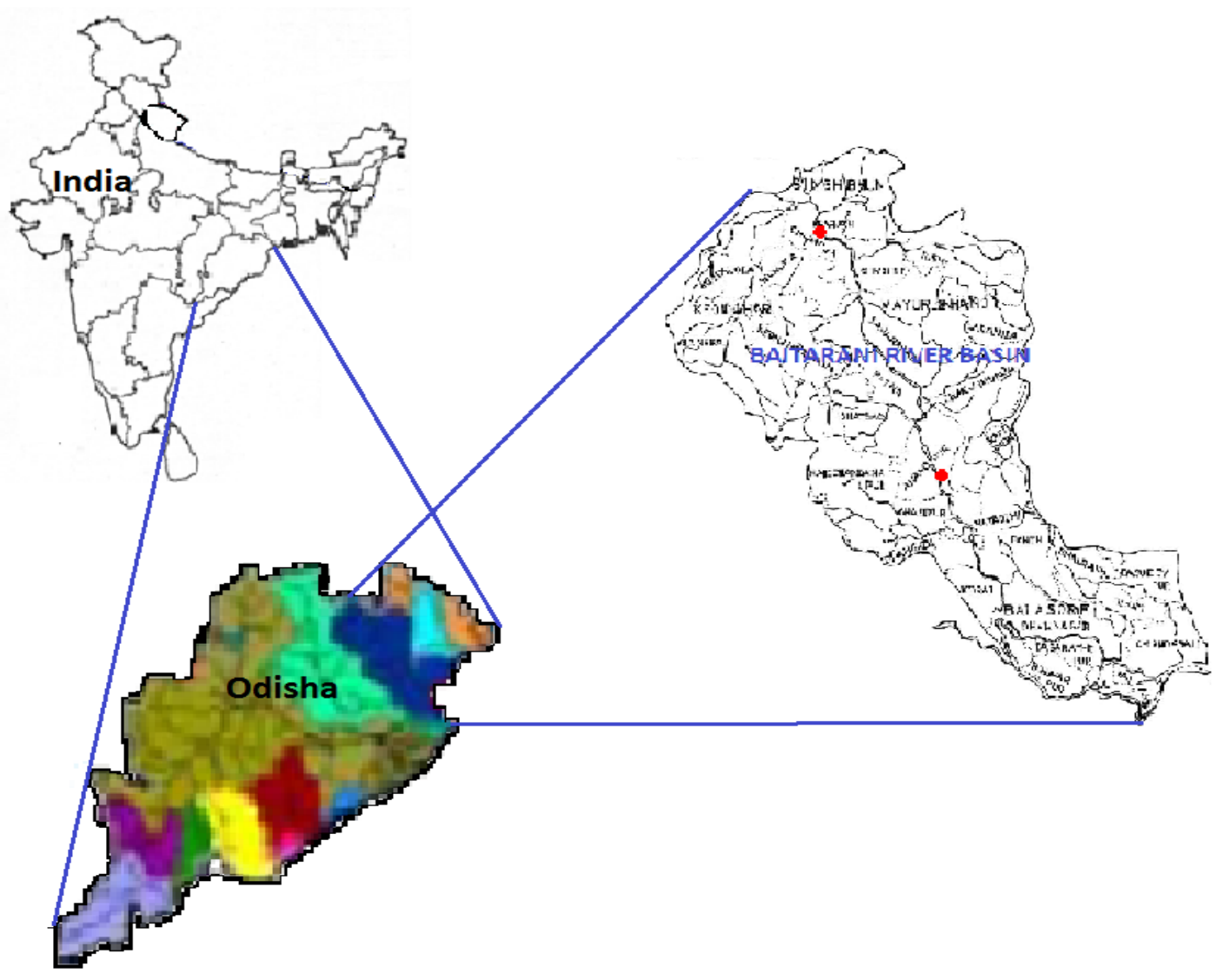


Fig.2 Daily SSC for monsoon season from 1977 to 2006 on Baitarani River

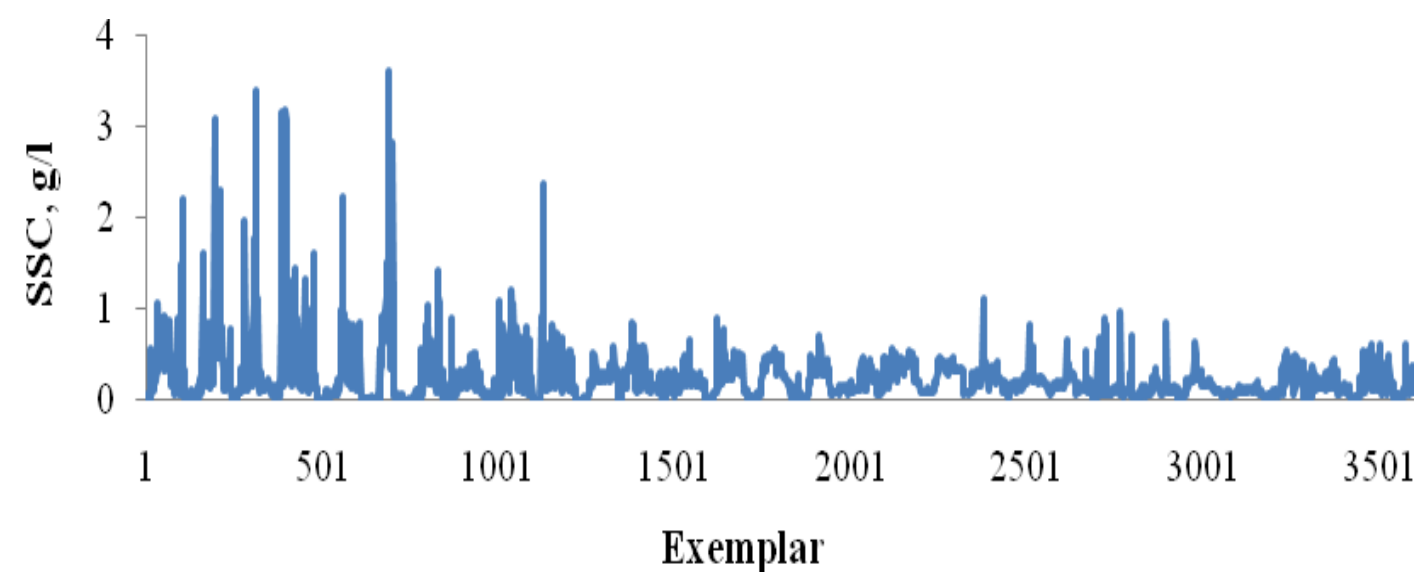

Fig.3 Daily discharge for monsoon season from 1977 to 2006 on Baitarani River

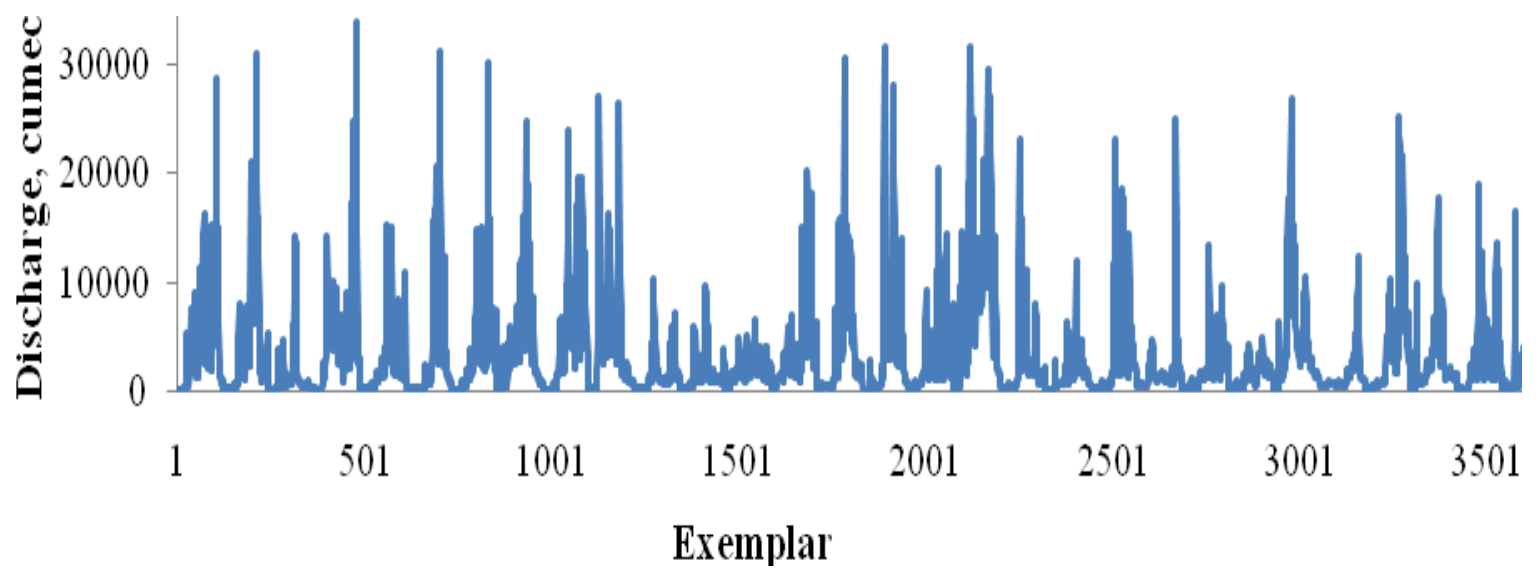

Fig.4 Structure of the perceptron

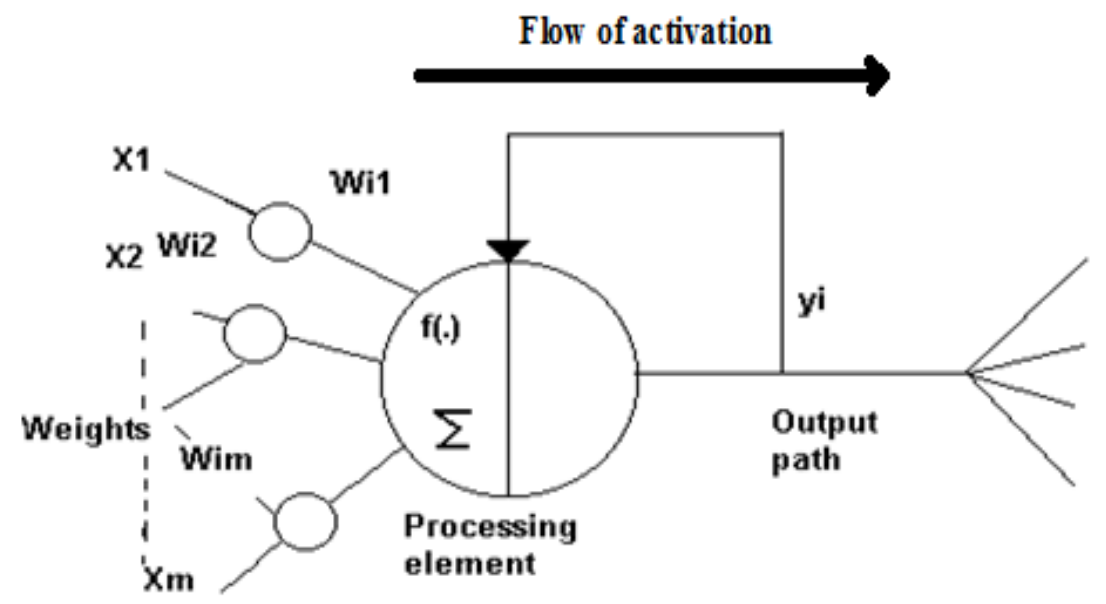


Fig.5 Observed (So) and computed (Sc) SSC from M-1 to Fig.6 Scatter plots of SSC from M-1 M-8 models during testing period

to M-8 models during testing period
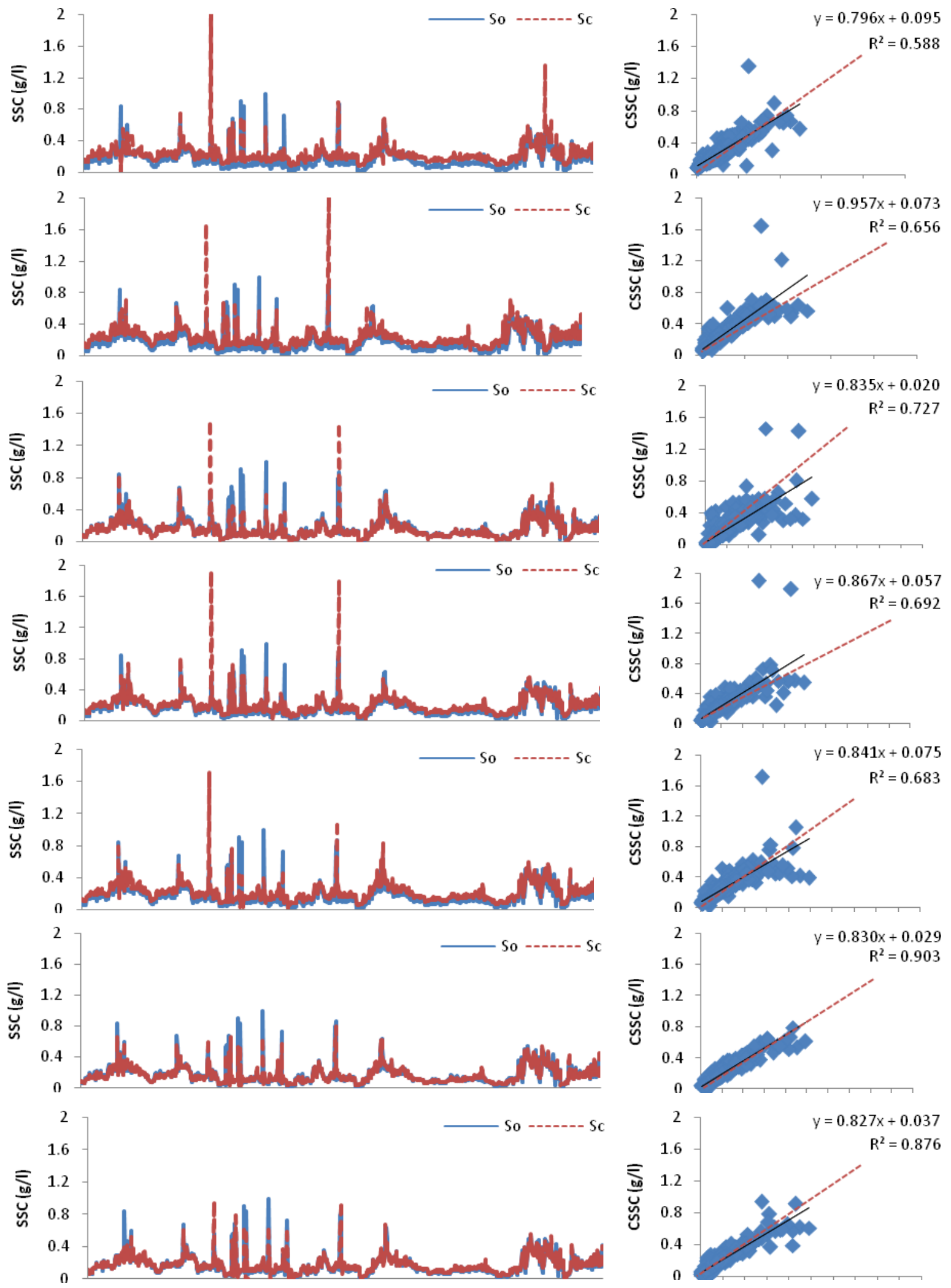

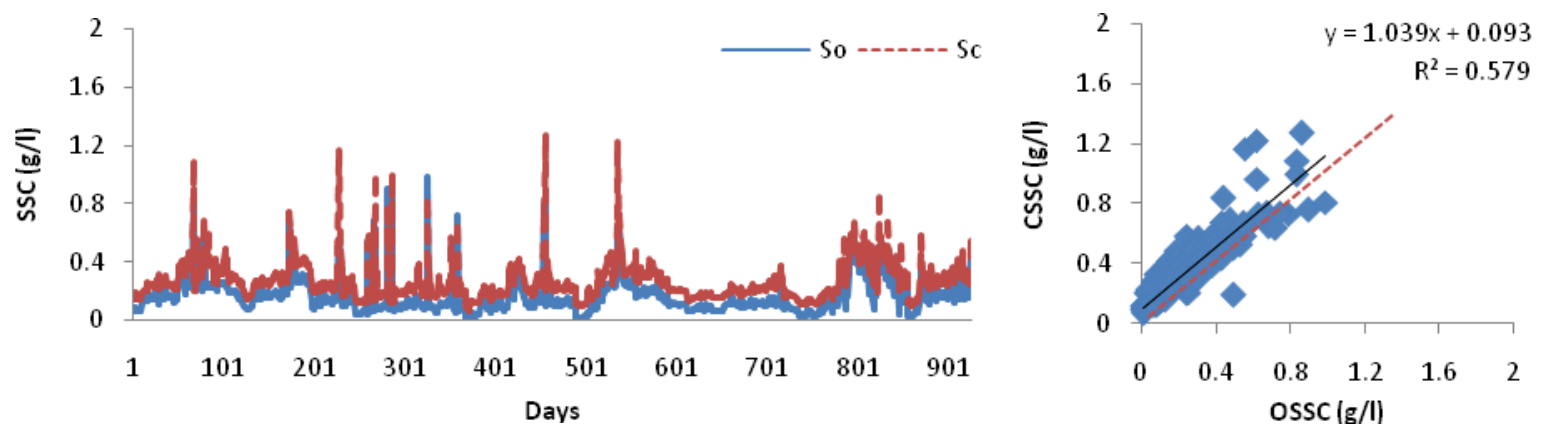

ANN-MLP

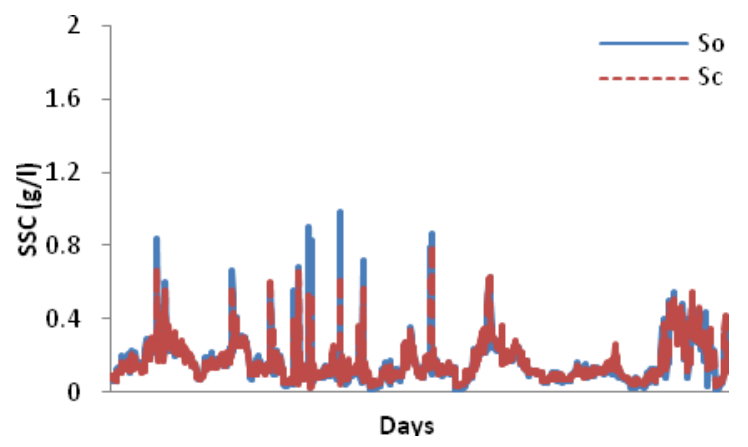

MLR

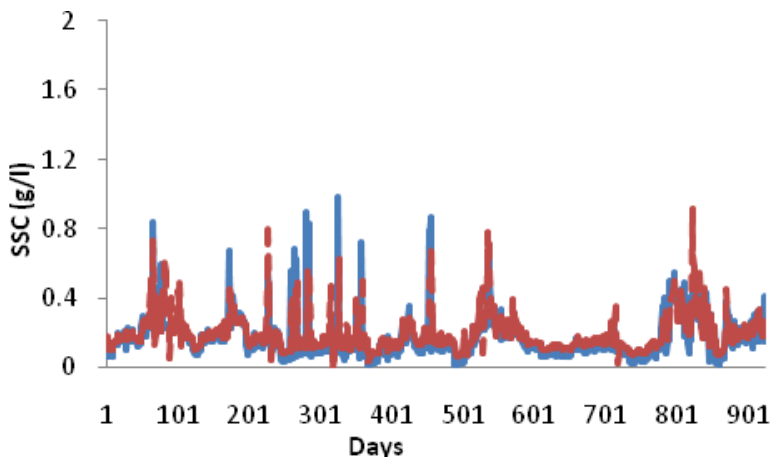

SRC

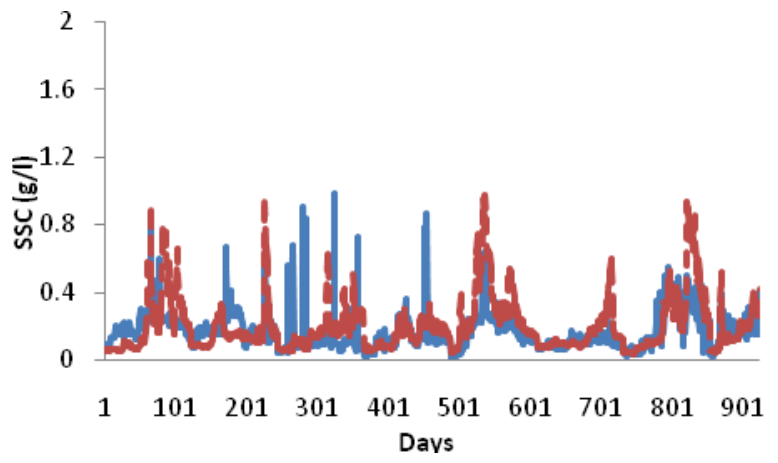

Fig.7 Observed (So) and computed (Sc) SSC of M-6 model during testing period
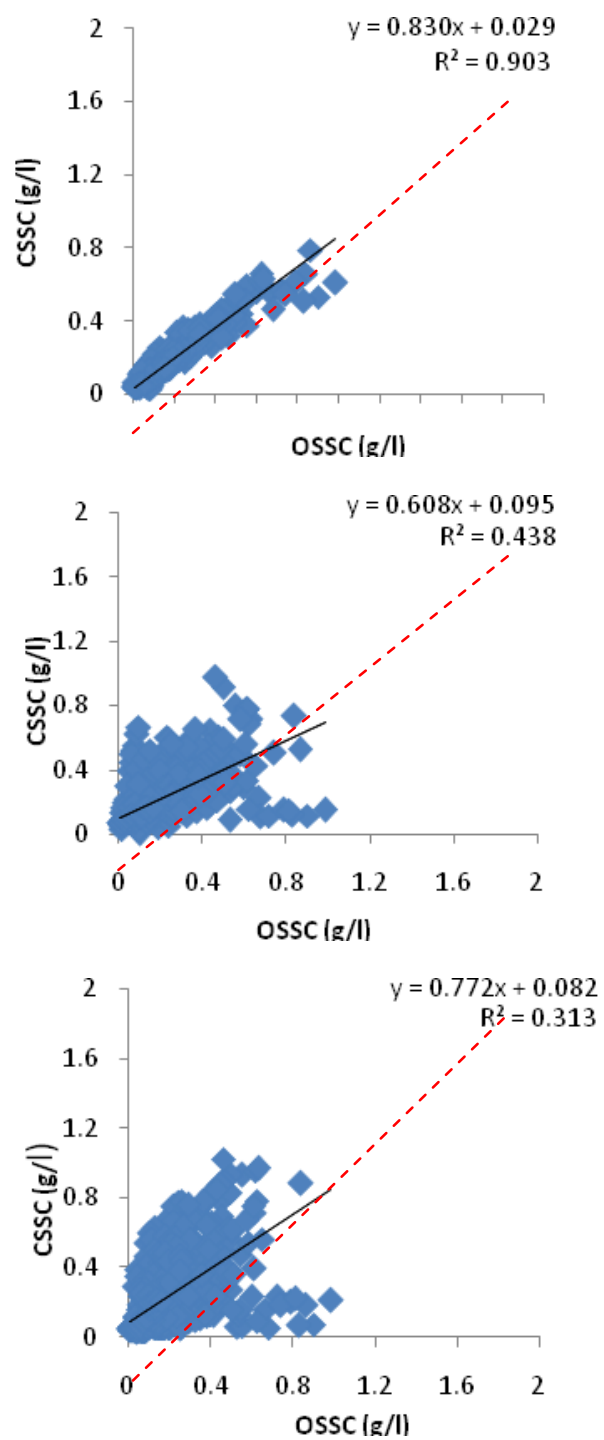

Fig.8 Scatter plots of SSC of M-6 model during testing period

It was observed from sediment hydrographs that out of eight models, M-3, M-6 and M-7 very closely predict the peaks accurately and rest of the models i.e. M-1, M-2, M-4, M-5 and M-8 over predict the peaks using ANNMLP method. 
In ANN-MLP method, the observations of scatter diagrams on the basis of best fit line and 1:1 line indicate that the SSC is over predicted for smaller values of SSC and under predicted for larger values of SSC using M-1, M-3, M-4, M-5, M-6 and M-7 models and over predicting for M-2 and M-8 models. It was also observed for M-3 model that most of the SSC values are under predicted and very few SSC values are over predicted.

M-6 and M-7 models nicely demonstrate that most of the data points are quite near the line of best fit in comparison to other models. But model M-7 shows some deviations from best fit line in comparison to M-6 model due to which performance of the M-7 model was found better than other models but inferior than M-6. Therefore, M-6 model was found to be better than other models for daily SSC prediction using ANN-MLP methods. The values of $R^{2}$ for models M-1, M-2, M-3, M-4, M-5, M-6, M-7 and M-8 are 0.588, 0.656, $0.727,0.692,0.6830 .903,0.876$ and 0.579 , respectively.

In Quantitative evaluation, the models having minimum values of RMSE, NMSE, MDL and AIC and higher values of CE and $r$ were considered as best models. Based on the selected criteria, five ANN based models i.e. M-3, M-4, M-5, M-6 and M-7 were found to be performing better than out of the eight models station.

Based on comparison among five selected models i.e. M-3, M-4, M-5, M-6 and M-7, M6 model had the minimum values of NMSE (0.1013), AIC (-11075.64), MDL (-10949.82) and RMSE (0.0100 g/l) and maximum values of CE (0.9937) and $r$ (0.951). The order of the models performance from best to worst was found to be M-6 > M-7 > M-3 > M-4> M-5. Therefore, performance of the M-6 model was found to be best in comparison to other models under ANN-MLP method.
It was also found that number of input variables is increasing with discharge in the river.

On the basis of comparison between qualitative and quantitative evaluation for best model, it was found that M-6 model in which present day SSC depends on the present day discharge, one-two and three lag days discharge, one-two and three lag days SSC with (7-5-5-1) network architecture i.e. 7 input variables, five-five neurons in first and second hidden layer and single output processing element is best out of eight models.

From the Comparison of M-6 model based on sediment hydrographs for ANN-MLP, MLR and SDR, it was observed from ANN-MLP based sediment hydrograph, that it is very closely predicting the peaks accurately out of three sediment hydrographs for MLR analysis sediment hydrograph and SRC sediment hydrograph because these are over predicting the peaks. It was also observed that sediment hydrograph of MLR analysis is giving better result than $\mathrm{SRC}$.

From the Comparison of M-6 model based on scatter plots for ANN-MLP, MLR and SDR, it was observed on the basis of best fit line and 1:1 line (dotted line) that the SSC is over predicted for smaller values of SSC and under predicted for larger values of SSC for all the methods applied in this study. It was also observed that most of the SSC values are under predicted and very few SSC values are over predicted for ANN-MLP.

M-6 model of ANN-MLP nicely demonstrates that most of the data points are quite near the line of best fit in comparison to other methods. Therefore, on the basis of qualitative comparison, ANN-MLP was found to be better than other methods for daily SSC prediction. The values of $R^{2}$ for ANN-MLP, 
MLR and SDR are 0.903, 0.438 and 0.313, respectively, for M-6 model.

Under quantitative comparison among ANNMLP, MLR and SDR for M-6 model, ANNMLP based M-6 model has the minimum value of RMSE $(0.0100 \mathrm{~g} / \mathrm{l})$ and maximum values of $\mathrm{CE}(0.9937)$ and $r(0.951)$. The order of the methods from best to worst was found to be ANN-MLP > MLR > SDR. Therefore, performance of the ANN-MLP based M-6 model was found to be best in comparison to other models.

On the basis of qualitative and quantitative comparison among ANN-MLP, MLR and SDR methods, ANN-MLP was found better than the others and ANN-MLP based M-6 model was found better than all the models and methods in this study.

Finally it was concluded that, ANN based SSC models can successfully be applied for the prediction of daily SSC at Champua station of Baitarani river.

\section{Acknowledgements}

We extend our appreciation to the Central Water Commission for providing useful data and G. B. Pant University of Agriculture and Technology for providing necessary facility of computer laboratory for this research work. We acknowledge Technical Education Quality Improvement Programme-II (201617) for funding the work.

\section{References}

ASCE. 2000a. Task Committee on application of ANN in hydrology, ANN in Hydrology, I: Preliminary Concepts. Journal of Hydrologic Engineering ASCE. 5(2), 124-137.

ASCE. 2000b. Task Committee on application of ANN in hydrology, ANN in Hydrology, II: Hydrologic Application. Journal of
Hydrologic Engineering ASCE. 5(2), 115-123.

Cobaner, M., Unal, B., Kisi, O., 2009. Suspended sediment concentration estimation by an adaptive neuro-fuzzy and neural network approaches using hydro meteorological data. Journal of Hydrology. 367(1-2), 52-61.

Danh, N.T., Phien, H.N., Gupta, A.D., 1999. Neural network models for river flow forecasting. Water S.A. 25 (1), 33-39.

Dawson, C.W., Wilby, R.L., 1998. An ANN approach to rainfall runoff modeling. Hydrological Sciences Journal. 43(1), 47-66.

Eisazadeh, L.L., Sokouti, R., Homoaee, M., Pazira, E., 2013. Modelling sediment yield using ANN and multiple linear regression methods. International Journal of Bioscience. 3(9), 116-122.

Elshorbagy, A., Simonovic, S.P., Panu, U.S., 2000. Performance evaluation of ANN for runoff prediction. Journal of Hydraulic Engineering. 5 (4), 424-427.

Gharde, K.D., Kothari, M., Mittal, H.K., Singh, P.K., Dahiphale, P.A., 2015. Sediment Yield Modelling of Kal River in Maharashtra Using ANN Model. Res. Journal Recent. Science. 4, 120-130.

Ghorbani, M.A., Hosseini, S.H., Fazelifard, M.H., Abbasi H., 2013. Sediment load estimation by MLR, ANN, NF and Sediment Rating Curve (SRC) in Rio Chama River. Journal of Civil Engineering and Urbanism. 3(4), 136-141.

Imrie, C.E., Durucan, S., Korre, A., 2000. River flow prediction using ANN: generalization beyond the calibration range. Journal of hydrology. 233 (1-4), 138-153.

Jain, S., 2008. Development of integrated discharge and sediment rating relation using a compound neural network. Journal of Hydraulic Engineering. 13(3), 124-131.

Jain, S.K., 2001. Development of integrated sediment rating curves using ANN. Journal of Hydrologic Engineering. ASCE 127(1), 3037.

Khoob, A.R., 2008. ANN estimation of reference evapotranspiration from pan evaporation in a semi-arid environment. Irrigation Science. 27 (1), 35-39.

Kisi, O., 2010. River suspended sediment concentration modeling using a neural differential evolution approach. Journal of 
Hydrology. 389, 227-235.

Kisi, O., Dailr A.H., Cimen, M., Shiri, J., 2012. Suspended sediment modeling using genetic programming and soft computing techniques. Journal of Hydrology. 450 - 451, 48-58.

Kisi, O., Shiri, J., 2012. River suspended sediment estimation by climatic variables implication: Comparative study among soft computing techniques. Computers \& Geosciences. 43, 73-82.

Kumar, A.R.S., Ojha, C.S.P., Goyal, M.K., Singh, R.D., Swame, P.K., 2011. Modeling of Suspended Sediment Concentration at Kasol in India Using ANN, Fuzzy Logic, and Decision Tree Algorithms. Journal of Hydrologic Engineering. 17, 394-404.

Kumar, D., Pandey, A., Sharma, N., Flügel, W., 2016. Daily suspended sediment simulation using machine learning approach. Catena. 138, 77-90.

McCulloch, W., Pitts, W., 1943. A Logical Calculus of the Ideas Immanent In Nervous Activity. Bulletin of Mathematical Biophysics. 5,115-133.

Nayak, P.C., Sudheer, K.P., Rangan, D.M., Ramasastri, K.S., 2004. A neurofuzzy computing technique for modelling hydrological time series. Journal of Hydrology. 291, 52-66.

Olyaie, E., Banejad, H., Chau, K.W., Melesse, A.M., 2015. A comparison of various artificial intelligence approaches performance for estimating suspended sediment load of river systems: a case study in United States. Environment Monitoring Assessment. 187, 189.

Principe, J.C., Euliano, N.R., Lefebvre, W.C., 2000. Neural and adaptive systems: fundamentals through simulations. John Wiley
\& Sons Inc., New York.

Rai, R.K., Mathur, B.S., 2008. Event- based Sediment Yield Modeling using ANN. Water Resource Manage. 22, 423-441.

Rajaee, T., Mirbagheri, S.A., Zounemat-Kermani, M., Nourani, V., 2009. Daily suspended sediment concentration simulation using ANN and neuro-fuzzy models. Science Total Environment. 407, 4916-4927

Sarangi, A., Bhattacharya, A.K., 2005. Comparison of ANN and regression models for sediment loss prediction from Banha watershed in India. Agricultural Water Management. 78(3), 195-208.

Shabani, M., Shabani, N., 2012. Estimation of daily suspended sediment yield using ANN and sediment rating curve in Kharestan Watershed, Iran. Australian Journal of Basic and Applied Sciences. 6, 157-164.

Shirsath, P.B., Singh, A.K., 2010. A comparative study of daily pan evaporation estimation using ANN, regression and climate based models. Water Resource Management. 24, 1571-1581.

Singh, A., Imtiyaz, M., Isaac, R.K., Denis, D. M., 2013. Comparison of ANN Models for Sediment Yield Prediction at Single Gauging Station of Watershed in Eastern India. Journal of Hydrologic Engineering. 18, 115-120.

Solaimani, K., 2009. Rainfall-runoff Prediction Based on ANN (A Case Study: Jarahi Watershed). American-Eurasian Jornal of Agriculture and Environment Science. 5 (6), 856-865.

Zounemat-Kermani, M., Kisi, O., Adamowski, J., Ramezani-Charmahineh, A., 2016. Evaluation of data driven models for river suspended sediment concentration modeling. Journal of Hydrology. 535, 457-472.

\section{How to cite this article:}

Daniel Prakash Kushwaha and Devendra Kumar. 2017. Suspended Sediment Modeling with Continuously Lagging Input Variables Using Artificial Intelligence and Physics based Models. Int.J.Curr.Microbiol.App.Sci. 6(10): 1386-1399. doi: https://doi.org/10.20546/ijcmas.2017.610.164 